\title{
Objective assessment of vitiligo with a computerised digital imaging analysis system
}

\begin{abstract}
An objective tool to quantify treatment response in vitiligo is currently lacking. This study aimed to objectively evaluate the treatment response in vitiligo by using a computerised digital imaging analysis system (C-DIAS) and to compare it with the physician's global assessment (PGA). Tacrolimus ointment 0.1\% (Protopic; Astellas Pharma Tech,Toyama, Japan) was applied twice daily on selected lesions which were photographed every 6 weeks for 24 weeks. The primary efficacy end-point was the mean percentage of repigmentation (MPR), as assessed by the digital method (MPR-C-DIAS) or by the PGA. The response was categorised into none $(0 \%)$, mild (1-25\%), moderate (26-50\%), good $(51-75 \%)$ and excellent (76-100\%). MPR-C-DIAS: Out of 56 patients, 44 (79\%) responded. Overall, the response was mild in $22(39 \%)$, moderate in $21(40 \%)$ and good in one (2\%) patient(s). A total of $39(70 \%)$ patients responded as measured by PGA. The repigmentation was mild in $27(48 \%)$, moderate in $10(18 \%)$ and good to excellent in two $(4 \%)$ patients. The $\kappa$ test of consistency was $0.17(\mathrm{P}=0.053)$, which shows poor agreement between the two assessment methods, although this is not statistically significant. The C-DIAS can be used to perform an objective analysis of repigmentation or depigmentation in vitiligo skin lesions in response to treatment.
\end{abstract}

Keyword: Assessment method; Vitiligo; Objective; Computerised; Digital imaging analysis 